\title{
Corela
}

Cognition, représentation, langage

HS-22 | 2017

Prédication et prépositions en anglais

\section{Les prépositions en contexte}

Approche de la théorie des opérations prédicatives et énonciatives (TOPE)

Jean-Marie MERLE

\section{(2) OpenEdition}

\section{Journals}

Édition électronique

URL : http://journals.openedition.org/corela/4973

DOI : $10.4000 /$ corela.4973

ISSN : $1638-573 \mathrm{X}$

Éditeur

Cercle linguistique du Centre et de l'Ouest - CerLICO

Référence électronique

Jean-Marie MERLE, "Les prépositions en contexte », Corela [En ligne], HS-22 | 2017, mis en ligne le 05 septembre 2017, consulté le 20 avril 2019. URL : http://journals.openedition.org/corela/4973 ; DOI : $10.4000 /$ corela.4973

Ce document a été généré automatiquement le 20 avril 2019

\section{(c) (i) (2)(2)}

Corela - cognition, représentation, langage est mis à disposition selon les termes de la licence Creative Commons Attribution - Pas d'Utilisation Commerciale - Partage dans les Mêmes Conditions 4.0 International. 


\section{Les prépositions en contexte}

Approche de la théorie des opérations prédicatives et énonciatives (TOPE)

Jean-Marie MERLE

\section{Introduction}

1 Cet article traite des prépositions selon l'approche énonciative en s'appuyant sur le corpus fourni par l'extrait de The Blind Assassin, de Margaret Atwood (p. 3-5). On commencera par quelques observations sur les propriétés de la préposition (\$1-3) - sa position, la relation prépositionnelle -, ce faisant, on reviendra sur ses définitions ( $\$ 1)$, en envisageant le statut nominal de l'élément régi par la préposition (\$2) et l'inclusion des particules adverbiales dans la catégorie de la préposition, à titre de prépositions monovalentes ( $\$ 3)$. Les deux caractéristiques saillantes de la préposition ne sont pas sa position ( $\$ 1.1$ ), mais 1 / sa fonction de relateur ( $\$ 1.2)$ - la relation prépositionnelle se caractérise comme la saisie d'une facette - facette d'un référent, d'un procès, d'un événement ou d'un état de fait $(\$ 4) ; 2$ / la relation de repérage qui accompagne la saisie de cette facette et qui caractérise la fonction prépositionnelle. La TOPE (Théorie des opérations prédicatives et énonciatives) est aussi nommée par Culioli «Théorie des repérages» (1990-1999). Cette relation de repérage est de trois ordres différents: repérage structurel, par incidence $(\S 5)$; repérage énonciatif, par indexation $(\$ 5)$; repérage sémantico-référentiel ( $(6)$.

\section{La préposition : propriétés}

\subsection{Préposition et position}

2 Le terme de préposition annonce une catégorie que l'on pourrait être tenté de définir par sa position. À la ligne 16, la préposition in se trouve effectivement placée avant reality, position canonique dans la formation de la locution prépositionnelle in reality ; à la même 
ligne, la préposition from se trouve en position initiale dans le syntagme prépositionnel from a distance.

Mais les prépositions dites orphelines (en anglais : stranded), c'est-à-dire placées en fin de proposition, sont courantes en anglais. On en trouve deux dans le texte :

1. (1. 25) [...] something [Ø she'd been locked up in]

2. (1. 28) What had she been thinking of [...]?

Dans l'exemple 1 (1.25), on a affaire à une relative: $\varnothing$ she'd been locked up in. Canoniquement, le relatif (ici, relatif Ø) est en position initiale. La préposition, quant à elle, ne peut se trouver entre l'élément complété et l'élément complément, puisque le premier (locked up) ne se trouve pas en amont du second (relatif Ø); c'est ainsi que la préposition reste orpheline après l'élément complété.

Dans l'exemple 2 (1.28), on a affaire à une structure interrogative directe en wh-, qui place en position initiale le pronom interrogatif what. La préposition of ne peut être à la fois derrière l'élément complété (thinking) et devant l'élément complément (what), pour la même raison que dans l'exemple précédent.

On a affaire dans les deux cas à une contrainte analogue sur la position de l'élément complément, et dans les deux cas la préposition se trouve orpheline.

La préposition ne peut donc se définir par sa position.

\subsection{Préposition et relation prépositionnelle}

8 La propriété qui caractérise la préposition, ce n'est pas sa position, mais sa fonction de relateur.

9 C'est cette fonction de mise en relation qui sous-tend la plupart des définitions proposées dans la littérature. En voici deux :

Le terme de relateur [...] répond au souci de recouvrir sous un concept englobant l'ensemble des éléments qui, selon les langues, sont soit des prépositions, soit des postpositions, soit des désinences casuelles, soit une combinaison de deux de ces moyens. (Hagège, $1997: 19$ )

In [Max sent a photograph of his new house to his parents], the preposition of relates the NP his new house to the noun photograph (we understand that the new house is depicted in the photograph), while to relates the NP his parents to the verb send (we understand his parents to have been the recipients of the photograph). (Huddleston \& Pullum, 2002 : 598)

Ainsi les énoncés suivants contiennent tous une mise en relation opérée par une préposition - mise en relation entre

- un verbe (ex. 3, fell),

- un adjectif (ex. 4, furious),

- un nom (ex. 5, hat),

- une proposition (ex. 6, they'd noticed her hands on the wheels)

et, respectivement

- the ravine (ex. 3), via la préposition into ;

- Laura (ex. 4), via la préposition with ;

- what she'd done (ex. 4) via la préposition for;

- a veil (ex. 5) via la préposition with ;

- the white gloves she'd been wearing via la locution prépositive because of (ex. 6).

3. (1. 2) The car fell a hundred feet into the ravine [...]

4. (1. 17-18) I was furious with Laura for what she'd done, [...] 
5. (1.34) [...] a hat with a veil.

6. (l. 11) They'd noticed her hands on the wheel because of the white gloves she'd been wearing.

11 En tant que relateur, dans ces quatre exemples, la préposition a un fonctionnement bivalent. Elle admet deux éléments dans sa valence, selon un schéma que l'on pourrait représenter - suivant Franckel \& Paillard (2007) - X Prép Y :

- X = fell / furious / hat / They'd noticed her hands on the wheel ;

- Prép = into / with; for / with / because of ;

- $\mathrm{Y}=$ the ravine / Laura ; what she'd done / a veil / the white gloves she'd been wearing.

\section{Le statut nominal du complément de la préposition, $\mathrm{Y}$}

Dans la relation X Prép Y, l'élément $Y$ est régi, syntaxiquement et sémantiquement, par la préposition. Ce complément $\mathrm{Y}$ a un statut nominal (cf. the ravine / Laura / a veil / the white gloves she'd been wearing), y compris lorsqu'il s'agit d'une proposition (what she'd done). La substitution par un syntagme nominal - dans l'exemple 4, ci-dessous, par some reason-de la proposition (dans l'exemple 4, what she'd done) régie par une préposition (ici la préposition for) est toujours possible, et met en évidence le statut nominal de cette proposition :

4. (1. 17-18) [...] furious with Laura for what she'd done, [...]

4 '. [...] furious with Laura for some reason, [...]

Cette fonction relationnelle propre à la préposition a une implication sémanticoréférentielle : l'élément $Y$ peut préexister à la relation prépositionnelle, quelle que soit la détermination de Y. Dans l'exemple 3, la détermination forte de the ravine reçoit une justification situationnelle, indépendante de la relation prépositionnelle. Dans l'exemple 7, l'antériorité référentielle ne fait aucun doute - le pont préexiste à l'événement - et elle n'a rien d'incompatible avec la détermination de a bridge, a marquant une détermination faible; en l'occurrence le référent de a bridge se comprend entre quelconque (un pont parmi d'autres) et non quelconque (un pont spécifique) :

7. (1. 1) [...] my sister Laura drove off a bridge.

15 Le statut nominal de $\mathrm{Y}$ permet d'établir une analogie ${ }^{1}$ entre la valence verbale et la valence prépositionnelle, analogie qui peut se mettre en évidence entre 8 et 8 ' comme entre 4 et $4^{\prime}$ :

8. (1. 7) He said the tires may have caught on a streetcar track [...]

8 ' He said a few words / something [...]

\section{Extension de la catégorie de la préposition}

L'extension de la catégorie de la préposition peut se poursuivre dans deux directions :

$1 /$ d'abord en annexant à la catégorie de la préposition des éléments qu'on considère souvent comme des conjonctions parce qu'ils régissent des propositions, comme after dans l'exemple 9 (l. 1), qui régit the war ended ; on peut opérer la même substitution (9') que précédemment (8' ou 4'), à l'aide d'un syntagme nominal (the end of the war) ; 2/ ensuite, à partir de l'observation que after admet également un fonctionnement 
monovalent, comme dans 9", en intégrant les particules adverbiales dans la catégorie de la préposition ${ }^{2}$ :

9. (l. 1) Ten days after the war ended, my sister Laura drove a car off a bridge.

9 ' Ten days after the end of the war, my sister Laura drove a car off a bridge.

9 " Ten days after, my sister Laura drove a car off a bridge. spatiale initiale: la police est accueillie au rez-de-chaussée. Ici encore, la préposition (selon une définition large de la catégorie de la préposition), ou la particule adverbiale (selon une définition étroite de la catégorie) apporte une télicité au mouvement exprimé par went, et la télicité s'accompagne d'inchoation (initiation d'une localisation nouvelle de $I$, état adjacent du mouvement).

Remarque 3. La tentation de pousser l'analogie entre la fonction prépositionnelle et la fonction verbale peut être grande. Franckel \& Paillard (2007: 18) défendent cette position :

Dans tous les cas, la préposition a une fonction prédicative, y compris dans le cas où $\mathrm{X}$ [dans XPrépY] ne correspond pas à un terme directement identifiable dans l'énoncé. 

autour du verbe. Par ailleurs, fall étant un verbe de mouvement, le déplacement dans l'espace peut avoir une origine et une destination, qui font partie des arguments potentiels du verbe, et ne sont pas des circonstants ${ }^{6}$. Autrement dit, le complément prépositionnel fait partie du prédicat. Sur le plan référentiel, cet énoncé représente (construit la référence à) un événement (borné à gauche et à droite) - une chute : l'aboutissement de la chute est un fragment de cet événement, et non une donnée périphérique ou disjointe ${ }^{7}$. Pour revenir enfin sur l'hypothèse de Franckel et Paillard citée ci-dessus, la relation prépositionnelle serait plutôt d'ordre coprédicatif, marquée par sa dépendance par rapport à la structure d'accueil (cf. § 2, ci-dessus, et § 5, ci-dessous) et son indexation sur une situation d'énoncé (cf. § 5). En l'occurrence, il s'agirait d'un coprédicat intégré dans le prédicat ${ }^{8}$.

\title{
4. Fonction référentielle de la relation prépositionnelle : facette d'un procès, d'un état de fait, d'un référent, d'un événement
}

\author{
3. (1. 2) The car fell a hundred feet into the ravine [...] \\ 4. (1. 17-18) I was furious with Laura for what she'd done, [...] \\ 5. (1.34) [...] a hat with a veil. \\ 6. (1. 11) They'd noticed her hands on the wheel because of the white gloves \\ she'd been wearing.
}

Les relations prépositionnelles des exemples 3-6 ont pour fonction référentielle ${ }^{9}$ de mettre en place une facette, respectivement d'un procès (ex. 3), d'un état de fait (ex. 4), d'un référent (ex. 5) et d'un événement (ex. 6).

Dans l'exemple 3 (1.2), la relation prépositionnelle construite autour de into est un fragment du prédicat fell a hundred feet into the ravine, comme on l'a vu au $\S 3^{10}$. Référentiellement, le prédicat renvoie à un procès; en tant que procès de mouvement, il peut inclure dans ses arguments une référence à l'origine du mouvement, en l'occurrence de la chute, ainsi qu'une référence à la trajectoire du mouvement (into the ravine, trajectoire quantifiée par a hundred feet et complément de fell) et à son aboutissement. C'est ainsi que la relation prépositionnelle met en place l'une des facettes du procès, en l'occurrence la trajectoire et l'aboutissement de la chute.

Dans l'exemple 4 (1. 17-18, I was furious with Laura for what she had done), énoncé d'un état de fait (propriété transitoire I was furious), les deux relations prépositionnelles, construites respectivement autour de with et autour de for, mettent en place deux facettes de l'état de fait, représentées par les deux compléments prépositionnels de l'adjectif furious, l'une concernant la cible (et le déclencheur) de la fureur (furious with Laura), l'autre concernant sa cause (furious for what she had done).

Dans l'exemple 5 (l. 34, a hat with a veil), la relation prépositionnelle construite autour de with (with a veil) met en place une facette, une propriété saillante, à fonction référentielle restrictive et définitoire, du référent de a hat. 

been wearing), c'est l'événement they'd noticed her hands on the wheel qui est mis en relation avec l'une de ses facettes - l'une de ses circonstances, la cause, en l'occurrence - via because of ${ }^{11}$.

\section{Deux modes de repérage : repérage structurel, par incidence (Prép $Y \underline{\underline{60}} \mathrm{X}$ ) ; repérage énonciatif, par indexation ([Prép $\mathrm{Y} \underline{\underline{65}} \mathrm{E} X] \underline{\underline{60}} \underline{\underline{60}}$ Sit)}

La mise en place par la relation prépositionnelle de la facette $Y$ d'un procès, d'un état de fait, d'un référent ou d'un événement que l'on vient d'observer est un phénomène référentiel (cf. note $9, \S 4$ ). Cette facette $Y$ entre en relation avec l'élément $X$ dont elle est un fragment selon une relation d'incidence (Prép Y est incident à X), autrement dit selon une relation de repérage structurel. Le repérage structurel, par incidence de Prép Y à X, met en place une relation de dépendance et un repérage énonciatif par indexation de la relation prépositionnelle X Prép Y sur la situation d'énoncé qui inclut $X$.

3. (1. 2) The car fell a hundred feet into the ravine [...]

4. (1. 17-18) I was furious with Laura for what she'd done, [...]

5. (1. 34) [...] a hat with a veil.

6. (1. 11) They'd noticed her hands on the wheel because of the white gloves she'd been wearing.

Dans l'exemple 3, into the ravine est une facette du procès représenté par le prédicat fell $a$ hundred feet into the ravine, comme on l'a vu au $\S 4$. En sa qualité de complément directionnel, into the ravine est incident au noyau verbal fell. C'est cette relation orientée d'apport (le complément directionnel into the ravine) à support (le verbe de mouvement fell) qui constitue le repérage structurel, de Y par rapport à X.

Into the ravine a les caractéristiques des formes à mode non fini : la relation prépositionnelle est dépendante d'une structure d'accueil. Cette structure d'accueil est l'énoncé the car fell a hundred feet into the ravine et into the ravine est indexé sur la situation d'énoncé. L'indexation tient à ce que le complément prépositionnel représente l'aboutissement d'un événement, borné à gauche et à droite, mais dont la spécification n'est pas complète, ce qui implique des variables, c'est-à-dire une certaine plasticité : d'abord l'indexation de cet événement sur les événements qui l'encadrent dans la successivité du récit (she went right through the Danger sign [...] then the car burst into flames ); mais également l'indexation sur cet événement du contenu coprédicatif du syntagme participial smashing through the treetops, lui-même incident à son support the car. Le repérage énonciatif de $<$ Prép $Y>$ est donc un repérage par indexation de $<$ Prép $Y>$ sur la situation d'énoncé propre à la structure d'accueil.

Dans l'exemple 4, with Laura et for what she had done sont l'un et l'autre incidents à l'adjectif furious, et c'est ce repérage structurel qui met ces deux compléments de l'adjectif (compléments qui sont à la fois compléments syntaxiques et compléments de sens) en relation avec l'état transitoire I was furious. Le repérage énonciatif par indexation sur la situation d'énoncé se fait à la faveur de cette relation d'incidence. L'indexation tient à ce que chacune des deux relations prépositionnelles n'a de pertinence qu'autant que la propriété I was furious est validée et pertinente. Autrement dit, les relations 
prépositionnelles sont indexées sur la situation d'énoncé et sur ses variables, via la relation d'incidence qui correspond à l'interprétation des repérages structurels.

Dans l'exemple 5, with a veil (1.34) est un modifieur (de caractérisation épithétique) de hat. En sa qualité de modifieur, il est incident à hat (comme l'est le déterminant a). Ce repérage structurel place with a veil dans la dépendance de a hat, et c'est cette relation de dépendance qui met en place l'indexation sur la situation d'énoncé (1. 33-34) I would need gloves, and a hat with a veil : le repérage énonciatif se fait toujours par indexation en raison de la multiplicité des variables en jeu (notamment le modifieur circonstant, to visit the morgue, la plurimodalité qui détermine l'énoncé, le caractère virtuel de la situation d'énoncé, son aspect prospectif et sa dépendance par rapport au contexte).

Dans l'exemple 6, (1.11) because of the white gloves she'd been wearing est un modifieur circonstant, incident à la relation ${ }^{12}<$ They-notice her hands on the wheel>. En tant que dépendant (un circonstant est toujours circonstant de ...), il est indexé sur la situation d'énoncé correspondant à They'd noticed her hands on the wheel, en l'occurrence sur la phase dynamique de l'événement plutôt que sur sa phase résultante.

La facette référentielle $\mathrm{Y}$ mise en place par la relation prépositionnelle X Prép Y est établie via deux relations de repérage, la première une relation de repérage structurel par incidence ( $\mathrm{Y}$ est complément de $\mathrm{X}$ ou modifieur de $\mathrm{X}$ ) ; la seconde, relation de repérage énonciatif mise en place à la faveur de la première, est une relation d'indexation de $\mathrm{X}$ Prép Y sur la situation d'énoncé dont fait partie $\mathrm{X}$.

\section{La relation prépositionnelle : une relation de repérage sémantico-référentiel de $X$ par rapport à $Y$}

41 Le $\$ 1.2$ met en évidence différentes relations prépositionnelles, selon le modèle X Prép Y. Dans le cadre de la TOPE ou "Théorie des repérages ", on décrit cette relation comme une relation de repérage de $\mathrm{X}$ par rapport à $\mathrm{Y}$. C'est le repérage sémantico-référentiel que l'on tend à privilégier, sans doute parce qu'il correspond à l'interprétation du sens construit lorsqu'on a affaire à un circonstant, le circonstant fournissant par son sémantisme un repère temporel, spatial, causal, etc.

9. (1. 1) Ten days after the war ended, my sister Laura drove a car off a bridge.

6. (l. 11) They'd noticed her hands on the wheel because of the white gloves she'd been wearing.

42 Ainsi dans l'exemple 9, Ten days after annonce l'exploitation des coordonnées temporelles de l'événement repère the war ended. Ten days after the war ended apporte une spécification temporelle à l'événement my sister Laura drove off, dont il est modifieur. La saisie opérée par after (cf. ci-dessus § 3) est une saisie selon un point de vue - c'est de ce point de vue que vient l'aspectualité propre à la relation prépositionnelle ${ }^{13}$ - qui ordonne les deux événements l'un par rapport à l'autre. Ten days after the war ended (Ten days after Y) sert de repère temporel à my sister Laura drove a car off a bridge (X). Autrement dit, un événement, the war ended, muni de ses propres coordonnées, sert de repère temporel à un autre événement, my sister drove a car off a bridge.

Dans l'exemple 6 (déjà rencontré au $\$ 1.2$ ), la locution prépositive because of annonce une relation sémantico-logique entre le repéré $\mathrm{X}$ et le repère causal $\mathrm{Y}$. Because of the white gloves she'd been wearing $(\mathrm{Y})$ sert de repère causal à tout le contenu propositionnel they'd noticed her hands on the wheel (X). 
13. (1. 36) I went into my dressing-room [...].

14. (1. 10) [...] Laura [...] had plunged off the bridge.

Dans les exemples 13 et 14 , went et plunged sont des verbes de mouvement, dont le sémantisme implique un déplacement - une origine, une trajectoire et un aboutissement : les deux verbes, went et plunged, remplissent leur fonction de noyau prédicatif, et chacun reçoit une spécification sémantique apportée respectivement par into my dressing-room (ex. 13) et off the bridge (cf. ci-dessus les remarques faites sur l'exemple 3, au $§ 4$ et au §5). Le mouvement est repéré dans le premier cas (ex. 13) par rapport à son aboutissement, my dressing-room $(\mathrm{Y})$, dans le second (ex. 14), par rapport à son origine, the bridge $(\mathrm{Y})$, repérage intraprédicatif qui s'opère selon le sémantisme de la relation prépositionnelle : into (1.36) marque l'accès à un état de fait nouveau, en l'occurrence la localisation du référent de I dans my dressing-room ; off (1.10) marque un repérage en rupture par rapport à the bridge, et inclut dans le procès un état nouveau, la perte de contact avec le repère the bridge.

15. (1. 3) [...] my entire face was rigid with pain.

4. (1. 17-18) I was furious with Laura for what she'd done, [...]

Dans les exemples 15 et 4, ci-dessus, on a affaire à des prédicats adjectivaux : rigid (l. 3), et furious (1.17). L'adjectif ne remplit que la fonction prédicative et c'est la copule be, porteuse de temps, qui remplit la fonction nodale et modale.

La propriété $\mathrm{X}$, be rigid, dans l'exemple 15, est repérée sémantiquement par rapport à pain , Y, selon une relation sémantico-logique d'effet (la propriété $S$ be rigid elle-même) à cause (pain).

La propriété be furious, dans l'exemple 4, est repérée sémantiquement par rapport à sa cible Laura, Y, et par rapport à sa cause what she'd done, Y'.

5. (1. 34) [...] a hat with a veil.

16. (l. 16) [...] the calmness of my own voice [...]

Dans l'exemple 5, a hat est modifié par with a veil. Le repérage de X, a hat, se fait par rapport à $Y$, a veil, qui définit un type particulier de chapeau.

9 Dans l'exemple 16, the calmness est complété par of my own voice. Le repérage de $\mathrm{X}$, the calmness, se fait par rapport à $Y$, my own voice, à l'intérieur d'une structure qui nominalise la relation <my own voice-be calm>. Autrement dit, la propriété calmness (notion nominale du troisième ordre, ou propriété nominalisée) est repérée par rapport au siège de la propriété my own voice.

0 Dans ces quatre séries d'exemples, les repérages sémantico-référentiels interviennent respectivement dans la délimitation d'événements (ex. 9 et 6), de procès (ex. 13 et 14), d'états de fait (ex. 15 et 4 ) et de référents (ex. 5 et 16).

\section{Conclusions}

La préposition a une fonction de relateur ( $\$ 2$ ), qu'elle ait un fonctionnement bivalent (définition traditionnelle de la préposition) ou monovalent (définition traditionnelle de la particule adverbiale ou définition étendue de la catégorie de la préposition; §3). La tradition admet que le rôle de la relation prépositionnelle S Prép Y est une relation de repérage, repérage sémantico-référentiel de $X$ par rapport à $Y(\$ 6)$. L'observation montre que le fonctionnement monovalent s'accompagne également d'une relation de repérage, repérage d'un état de fait saisi par rapport à un état de fait initial (\$ 3). 


\section{BIBLIOGRAPHIE}

Ciraud-Lanoue, P. (2013). Prépositions, préverbes et post verbes en anglais et en français. Le cas de away, off et out. Approche syntaxique et sémantique, Thèse de doctorat, Université de Poitiers.

Culioli, A. (1990-1999). Pour une linguistique de l'énonciation - 3 tomes, Paris / Gap : Ophrys.

Damourette, J. \& Pichon É. (1911-1940). Des mots à la pensée. Essai de grammaire de la langue française . 7 volumes, Paris : Editions d'Artrey 1936, réimp. 1970.

Dubois-Charlier, F. (2001). Compléments de Verbe, de Proposition, de Phrase, d'Enoncé, in Adverbe et circonstant, Travaux du CLAIX n ${ }^{\circ}$ 17, Aix-en-Provence : Publications de l'Université de Provence, 33-50.

Franckel, J.-J. \& Paillard D. (2007), Grammaire des prépositions, Tome 1, Paris : Ophrys. 
Hagège, C. (1997). Les relateurs comme catégorie accessoire et la grammaire comme composante nécessaire, in La préposition : une catégorie accessoire ?, Faits de Langues $\mathrm{n}^{\circ} 9$, Danon-Boileau L. \& Morel M.-A. (dir.), Paris / Gap : Ophrys, 19-27.

Huddleston, R. \& Pullum G. K. (2002). The Cambridge Grammar of the English Language, Cambridge University Press.

Huddleston, R. \& Pullum G. K. (2005). A Student's Introduction to English Grammar, Cambridge University Press.

Merle, J.-M. (2008). « Prépositions et aspect », L'information grammaticale, Leeman D. (dir.), n 117, 52-56.

Merle, J.-M. (2011). Remarques générales sur les prépositions en anglais, et sur into et out of en particulier, Travaux du CLAIX n² 21, Aix-en-Provence : Presses universitaires de Provence, 249-274.

Merle, J.-M. (2015). Présentation générale, Prépositions et aspectualité, Faits de Langues n 44 , Berne : Peter Lang, 5-12.

Wilmet, M. (1997a), Grammaire critique du français, Paris : Duculot.

Wilmet, M. (1997b). L'apposition : une fonction à réestimer, in Kleiber G. \& Riegel M. (dir), Les formes du sens. Etudes de linguistique française, médiévales et générales offertes à Robert Martin, Louvain-La-Neuve : Duculot, 413-422.

\section{Bibliographie complémentaire}

Busuttil, P. (2003). « Pouvoir absolu ou pouvoir partagé ? (Quelques réflexions sur le statut du deuxième terme des verbes complexes anglais) » in Les prépositions dans la rection des verbes (domaine anglais) CIRLEP. Presses Universitaires de Reims.

Cadiot, P. (1997). Les prépositions abstraites en français, Paris : Armand Colin. Jespersen, O. (1924). The Philosophy of Grammar, Londres, George Allen \& Unwin. Traduction française : Anne-Marie Léonard, 1971, La philosophie de la grammaire, Paris : Tel, Gallimard. Joly, A. \& O’Kelly D. (1990). Grammaire systématique de l'anglais, Paris : Nathan.

Langlais, A. (2000). « De BY en BY : « Airs de famille » d'une catégorie », Anglophonia 8, Toulouse : Presses Universitaires du Mirail.

Langlais, A. (2001). « De l'espace à l'aspect : over », Anglophonia 10, Toulouse : Presses Universitaires du Mirail, 185-198.

Larreya, P. \& Rivière C. (2005). Grammaire explicative de l'anglais, Paris : Pearson Longman.

Wyld, H. (1993). Since et les types de procès, Cahiers de recherche t. 6, Types de procès et repères temporels, Bouscaren J. \& Deschamps A. (dir.), Paris / Gap : Ophrys, 35-83.

Wyld, H. (2001). Subordination et énonciation, Paris / Gap : Ophrys.

\section{NOTES}

1. Cf. par exemple Damourette \& Pichon, 1911-1940, tome VII, § 2998-3003.

2. Cf. la position de Huddleston \& Pullum (2002). Pour une discussion de ce point, cf. Merle (2011).

3. De telos, borne, terme, limite: la télicité correspond à une borne de droite, terme de la complétude ou de l'achèvement. 
4. Sur la complémentarité de la télicité et de l'inchoation, voir Merle $2008: 52,55-56 ; 2011: 270$.

5. Cf. la thèse de Ciraud-Lanoue (2013).

6. Cf. Dubois-Charlier 2001. Deux tests de dissociation du syntagme prépositionnel restent négatifs : $1 /$ ? $^{*}$ The car fell into the ravine and so did the bus into the precipice; $2{ }^{*}$ The car fell and it did so into the ravine.

7. Les mêmes tests de reprise prédicative permettent d'opposer les arguments (qui appartiennent au prédicat) et les circonstants en français : *la voiture est tombée et ça s'est passé dans le ravin (test négatif car dans le ravin est un argument directionnel et non un circonstant) vs La voiture est tombée dans le ravin samedi soir à cause d'une rupture de la colonne de direction ; ça s'est passé samedi soir / La voiture est tombée dans le ravin et c'est arrivé à cause d'une rupture de la colonne de direction (tests positifs car « samedi soir » et « à cause d'une rupture de la colonne de direction " sont des circonstants et non des arguments).

8. Les coprédicats intégrés dans le prédicat s'opposent aux coprédicats détachés de la structure d'accueil : de manière prototypique, l'opposition entre intégration et détachement, pour ce qui est des coprédicats, est clairement illustrée, dans le cadre d'une terminologie standard, par les " attributs de l'objet » (coprédicats intégrés) et les "appositions » (coprédicats détachés). La notion d'apposition peut cependant recevoir une définition plus large (cf. Wilmet 1997a, 1997b), correspondant de façon générale au coprédicat.

9. La fonction référentielle des noms et des syntagmes nominaux est de représenter des référents, les prédicats représentent des procès, les relations prédicatives représentent états de faits et événements.

10. Into the ravine n'est pas circonstant comme le montrent les tests : $1 /{ }^{*}$ The car fell, and she/it did so a hundred feet into the ravine; $2{ }^{*}$ The car fell into the ravine, and so did the bike into the river.

11. Because of the white gloves she was wearing est un circonstant adjoint, modifieur de toute la relation they'd noticed her hands on the wheel. Tests : 1/ They'd noticed her hands on the wheel and they'd done so because of the white gloves she was wearing; 2/ They'd noticed her hands on the wheel because of the white gloves she was wearing and so had another witness because her hands were fidgety; 3/ It was because of the white gloves she was wearing that they'd noticed her hands on the wheel.

12. Le circonstant adjoint est typiquement incident à une relation d'incidence (cf. Wilmet 1997a)

13. Cf. Merle $2015: 5-12$.

\section{RÉSUMÉS}

Cet article traite des prépositions selon une approche énonciative. Il aborde les propriétés de la préposition (§ 1-3) et revient sur ses définitions ( $(1)$, sur le statut nominal de l'élément régi par la préposition ( $\$ 2$ ) et sur l'inclusion des particules adverbiales dans la catégorie de la préposition (§3). Les deux caractéristiques saillantes de la préposition sont 1 / sa fonction de relateur (§ 1.2) la relation prépositionnelle se caractérise comme la saisie d'une facette - facette d'un référent, d'un procès, d'un événement ou d'un état de fait (\$ 4);2/ la relation de repérage qui accompagne la saisie de cette facette et qui caractérise la fonction prépositionnelle. La TOPE (Théorie des opérations prédicatives et énonciatives) est aussi "Théorie des repérages ». Cette relation de repérage est de trois ordres différents : repérage structurel, par incidence (§ 5); énonciatif, par indexation (§5); repérage sémantico-référentiel (§ 6). 
This paper offers an enunciative approach to prepositions. The first section is about the general properties (§ 1-3) and possible definitions of prepositions ( $\S 1$ ). The second section examines the nominal and referential status of prepositional complements ( $\$ 2)$. The third section is about the inclusion of adverbial particles in the category of prepositions, and their general semantic, and aspectual properties ( $\$ 3)$. The two main properties of prepositions are $1 /$ their function as relators (\$1.2) - the prepositional relation represents a semantic facet of a referent (nominal phrase), a predicate, an event or a state of things ( $\$ 4) ; 2 /$ the orientations of the prepositional function as a relator in A Prep B. B is structurally (syntactically) located relative to A (§ 5); Prep B is enunciatively dependent and located relative to the embedding situation of utterance ( $\$ 5$ ); $A$ is semantically and referentially located relative to B (§6).

\section{INDEX}

Mots-clés : prépositions, relation prépositionnelle, particules adverbiales, saisie aspectuelle, repérage structurel, repérage énonciatif, repérage sémantico-référentiel

Keywords : prepositions, prepositional relation, adverbial particles, aspectual value, structural dependency, enunciative dependency, semantic and referential dependency

\section{AUTEUR}

\section{JEAN-MARIE MERLE}

Université Nice Sophia Antipolis

BCL (Bases, corpus, langage) (UMR7320),

jean-marie.merle@unice.fr 